\title{
Comorbilidades Psiquiátricas Associadas às Cefaleias: a Experiência da Consulta de Ligação
}

\author{
Psychiatric Comorbidities Associated with Headaches: the Experience of \\ the Liaison Consultation
}

\author{
Vera MARTINS'1, José TEMÓTIO'1, Ilda MURTA ${ }^{1}$ \\ Acta Med Port 2015 Jan-Feb;28(1):44-50
}

\section{RESUMO}

Introdução: A comorbilidade entre cefaleias e perturbações psiquiátricas é frequente, podendo ser explicada quer ao nível de modelos uni ou bidirecionais, quer ao nível da partilha de factores de risco genéticos e ambientais, comuns ao desenvolvimento de ambas as condições clínicas. A presença desta comorbilidade tem implicações importantes, agravando o quadro clínico, aumentando o risco de cronicidade, elevando a intensidade da dor e a taxa de insucesso terapêutico.

Material e Métodos: Os autores realizaram um estudo descritivo, retrospectivo, de prevalência, envolvendo 250 doentes observados na consulta de ligação de Psiquiatria-Cefaleias, durante um período de 3 anos, entre 1 de Julho de 2011 e 1 de Julho de 2013 , no qual se procedeu à análise dos processos clínicos, relativamente aos dados sociodemográficos e clínicos, diagnóstico psiquiátrico, neurológico e terapêutica prescrita.

Resultados e Discussão: Foram efectuadas 689 consultas de ligação Psiquiatria-Cefaleias, verificando-se maior prevalência de doentes do sexo feminino (84\%), com média de idades de 47 anos. As cefaleias tipo tensão $(60,8 \%)$, a enxaqueca $(24,8 \%)$ e as cefaleias atribuídas a perturbações psiquiátricas $(7,2 \%)$, foram os tipos mais prevalentes, enquanto as perturbações de humor (62\%) e as perturbações neuróticas relacionadas com o stress $(39,2 \%)$ foram os diagnósticos psiquiátricos mais frequentes na consulta. A intervenção terapêutica nestes casos privilegiou a intervenção multidisciplinar do médico Neurologista e do Psiquiatra, baseando-se na psicoeducação, abordagem cognitivo-comportamental e tratamento psicofarmacológico.

Conclusão: Dada a complexidade dos quadros clínicos nos casos de comorbilidade, a experiência da consulta de psiquiatria de ligação e da intervenção multidisciplinar revelou-se uma mais valia no tratamento destes doentes, configurando-se como o cenário mais adequado para o tratamento destas patologias.

Palavras-chave: Cefaleia; Comorbilidade; Perturbações Mentais; Psiquiatria.

\section{ABSTRACT}

Introduction: The comorbidity between headaches and psychiatric disorders is common and may be explained by different mechanisms in terms of uni or bi-directional models, or sharing of genetic and environmental risk factors relating to development of both clinical conditions. The presence of this comorbidity has important implications for patients with headaches and for patients with psychiatric disorders, worsens the clinical situation, increases the risk of chronicity, the pain intensity and the rate of treatment failure.

Material and Methods: The authors performed a descriptive, retrospective study of prevalence, involving 250 patients seen in Psychiatry - Headaches liaison consultation, over a period of 3 years, from July 1, 2011 to July 1, 2013. The authors undertook the analysis of the clinical records, in respect to sociodemographic and clinical data, neurological and psychiatric diagnosis and prescribed therapy.

Results and Discussion: During this period were made 689 liaison consultations, with a prevalence of female patients (84\%) and a mean age of 47 years. The tension type headache $(60.8 \%)$, migraine $(24.8 \%)$ and headache attributed to psychiatric disorders $(7.2 \%)$ were the most prevalent types of headaches. Mood disorders $(62 \%)$ and neurotic stress related disorders (39.2\%) were the most frequent psychiatric diagnoses. The therapeutic intervention in these cases emphasizes the multidisciplinary approach with the collaboration of Neurologist and Psychiatrist, based in psychoeducation, cognitive-behavioral intervention and psychopharmacological treatment.

Conclusion: Given the complexity of the clinical picture in cases of comorbidity, the experience of psychiatry liaison consultation and multidisciplinary intervention has proved particularly valuable in treating these patients, configuring itself as the proper treatment of this comorbidity.

Keywords: Comorbidity; Headache; Mental Disorders; Psychiatry.

\section{INTRODUÇÃO}

Segundo a Classificação Internacional de Cefaleias da Sociedade Internacional de Cefaleias (ICHD-3 beta), 1 estas podem ser divididas em Cefaleias primárias, ou cefaleias secundárias quando ocorrem pela primeira vez em estreita relação temporal com outra alteração que é uma causa reconhecida de cefaleias.

As cefaleias primárias compreendem a enxaqueca com ou sem aura, as cefaleias tipo tensão e a cefaleia em salvas, o tipo mais conhecido das cefaleias trigémino- autonómicas. As cefaleias secundárias podem ser devidas a situações de traumatismo crânio-encefálico e/ou cervical, doença vascular craniana ou cervical, perturbação intracraniana não vascular, uso de substâncias ou sua privação, infecção, perturbação da homeostase, perturbação de estruturas cranianas ou faciais e perturbações psiquiátricas. Por fim a parte 3 engloba as neuropatias cranianas dolorosas, outras dores faciais e outras cefaleias.

As cefaleias primárias são geralmente conceptualizadas

1. Centro de Responsabilidade Integrada de Psiquiatria e Saúde Mental. Centro Hospitalar e Universitário de Coimbra. Coimbra. Portugal.

Recebido: 29 de Maio de 2014 - Aceite: 17 de Setembro de 2014 | Copyright @ Ordem dos Médicos 2015 
como doenças crónicas, não sendo apenas episódios recorrentes de cefaleia aguda, mas condições dinâmicas que podem progredir ou remitir, com marcadores estruturais e neurorradiológicos bem identificados. Apesar de não estar identificado nenhum biomarcador patognomónico da enxaqueca, vários estudos imagiológicos mostraram alterações a nível do fluxo sanguíneo cerebral. Durante a crise de enxaqueca com os sintomas típicos de aura foi observada uma redução regional do fluxo sanguíneo cerebral (oligoemia), sobretudo nas regiões cerebrais posteriores, enquanto que durante a enxaqueca sem aura foi descrito um aumento do fluxo cerebral no tronco cerebral medial, predominantemente contralateral à cefaleia e no córtex cingulado, auditivo e visual. Estudos com ressonância magnética mostraram um aumento da incidência de lesões na substância branca em doentes com enxaqueca. ${ }^{2}$

Como se tratam de doenças neurobiológicas, as alterações neuropsiquiátricas não são inesperadas. A comorbilidade entre cefaleias e perturbações psiquiátricas é frequente, podendo ser explicada através de diferentes mecanismos, quer ao nível de modelos uni ou bidirecionais, quer ao nível da partilha de factores de risco genéticos e ambientais comuns ao desenvolvimento de ambas as condições clínicas. A ocorrência desta comorbilidade psiquiátrica tem implicações no tratamento dos doentes com cefaleias, impondo limitações e criando oportunidades que permitem conhecer os mecanismos subjacentes à fisiopatologia das cefaleias e da comorbilidade, com base em factores de risco partilhados por ambas as condições. ${ }^{3}$

É necessário um elevado grau de suspeição em doentes com cefaleias para diagnosticar as comorbilidades psiquiátricas, implicando a utilização de instrumentos para o despiste destas patologias, como várias escalas de depressão ou ansiedade de aplicação rápida, como a Escala de Ansiedade Generalizada (GAD-7) ou o Questionário sobre a Saúde do Paciente (PHQ-9).

As comorbilidades psiquiátricas relacionam-se com a progressão e agravamento das cefaleias, tratamento e resultados clínicos, sabendo-se que, por exemplo, a depressão está associada ao agravamento das cefaleias, nomeadamente à transformação de episódios de enxaqueca agudos em crónicos, e resultados negativos, como a existência de tentativas de suicídio. ${ }^{4}$ A comorbilidade psiquiátrica também tem impacto conhecido em termos de uma maior incapacidade e pior qualidade de vida relativamente a doentes com cefaleias sem comorbilidade psiquiátrica, ${ }^{5}$ pelo que o seu reconhecimento e tratamento tem particular importância.

As perturbações depressivas e ansiosas são, sem dúvida, as comorbilidades psiquiátricas mais frequentes em todos os tipos de cefaleias primárias e vários estudos têm demonstrado a sua prevalência aumentada, nomeadamente em termos de cuidados de saúde terciários.

As cefaleias tipo tensão são as cefaleias primárias mais frequentes, com prevalências na ordem dos 30 a $78 \%$ ao longo da vida, ${ }^{1}$ sendo frequente a sua comorbilidade com perturbações depressivas e de ansiedade dada a associa- ção conhecida com factores de stress.

No entanto, pelas características clínicas e pela incapacidade que provoca, a enxaqueca é a principal causa neurológica de procura de cuidados médicos, sendo frequente ao nível dos cuidados de saúde primários. ${ }^{6}$ Cerca de $1 / 3$ dos doentes que recorrem a este nível a cuidados de saúde apresentam critérios para enxaqueca ${ }^{7}$ e uma grande proporção apresenta alguma perturbação psiquiátrica em comorbilidade, nomeadamente nos casos de enxaqueca crónica. A enxaqueca crónica define-se pela presença de cefaleia ocorrendo em 15 ou mais dias por mês, durante mais de 3 meses, com características de cefaleia de enxaqueca, em pelo menos 8 dias por mês. ${ }^{1}$

Alguns estudos sugerem que as perturbações de humor e ansiedade são cerca de 2 a 10 vezes mais prevalentes em pessoas com enxaqueca relativamente à população geral e que cerca de $25 \%$ destas pessoas preenchem critérios para perturbação de humor e ansiedade. ${ }^{8,9}$

A prevalência das perturbações depressivas em pessoas com enxaqueca difere de acordo com os estudos e metodologia utilizada, variando entre os $4,3 \%$ e $47 \%$, sendo cerca de 2 vezes mais frequente nos casos de enxaqueca crónica do que episódica. ${ }^{10} \mathrm{~A}$ depressão é um dos principais factores de risco que contribui para a cronificação da enxaqueca, mas existem outros, tais como o abuso de medicação, a obesidade, as perturbações do sono ou outras comorbilidades psiquiátricas. Este efeito é dependente da intensidade da sintomatologia depressiva, o aumento da gravidade leva a maior risco de cronificação, sendo que o contrário não é válido, não havendo evidência de que a melhoria da sintomatologia depressiva interfira na remissão dos episódios crónicos para agudos, ${ }^{11}$ no entanto, a redução da frequência das cefaleias pode melhorar a sintomatologia depressiva e ansiosa.

Breslau et al $^{12}$ mostraram uma relação bidirecional entre depressão e enxaqueca, em que a depressão prediz o início de novo de enxaqueca e a enxaqueca aumenta a incidência de depressão major. No entanto, para os outros tipos de cefaleias primárias, apenas foi mostrada uma relação unidirecional em que as cefaleias aumentam a incidência de depressão, mas a depressão não prediz o início de novo de cefaleias, excepto enxaqueca. Estas evidências permitem concluir que os mecanismos de comorbilidade são diferentes consoante o tipo de cefaleias.

As perturbações de ansiedade, incluindo a Perturbação de Ansiedade Generalizada (PAG), a Perturbação de Pânico, a Perturbação Obsessivo Compulsiva (POC) e as fobias específicas, são cerca de duas vezes mais prevalentes em comorbilidade com a enxaqueca do que na população geral. Cerca de 50,7 a $57 \%$ de pessoas com enxaqueca têm perturbações de ansiedade, comparativamente com $27 \%$ da população geral, sem enxaqueca. ${ }^{8} \mathrm{~A}$ prevalência de PAG comórbida com enxaqueca varia entre duas a cinco vezes mais do que a população geral, consoante os estudos, e a perturbação de pânico pode ser três vezes mais prevalente em pessoas com enxaqueca. ${ }^{8}$ As perturbações de ansiedade são também consideradas como factor 
de risco para a cronificação dos episódios de enxaqueca, contribuindo para o seu agravamento em $70 \%$ dos casos, sendo que as perturbações do sono apenas em $22 \%$ dos casos foram identificadas como factor de agravamento das crises de enxaqueca. ${ }^{10}$

A enxaqueca tem sido também associada a Perturbações da Personalidade, Perturbação de Stress Pós Traumática (PSPT) e abuso de substâncias, nomeadamente em contexto de depressão ou PSPT. 8,9

A PSPT ocorre em cerca de $25 \%$ dos doentes com cefaleias, nomeadamente diárias e de carácter crónico, podendo ter como causa maus tratos na infância, episódios de violência ou outros eventos traumáticos. $\mathrm{O}$ abuso físico e sexual está associado a enxaqueca, provavelmente mediado por sintomatologia ansiosa, depressiva ou traumática, sendo o abuso emocional um factor reconhecido na cronificação da enxaqueca. ${ }^{10}$

A cefaleia em salvas é caracterizada por crises de dor associadas a sintomas autonómicos parassimpáticos proeminentes. A prevalência das perturbações depressivas em doentes com este tipo de cefaleias primárias varia entre os $6,3 \%$ e os $43 \%$ em estudos transversais e entre $4,6 \%$ e $56,6 \%$ em estudos de prevalência ao longo da vida, ${ }^{13}$ sendo maior nas formas de carácter crónico. Esta prevalência é semelhante ou mesmo inferior à que se verifica nos doentes com cefaleias de tensão.

Segundo Gestelgyi et al, ${ }^{14}$ a incapacidade gerada pela presença de dor revelou-se o preditor independente mais importante na gravidade dos sintomas depressivos em todos os subgrupos de doentes com cefaleias primárias (tendo sido omitido da análise o subgrupo da cefaleia em salvas devido ao pequeno número de casos $(n=11))$, contrariamente à intensidade da dor que não mostrou um carácter preponderante neste processo.

A prevalência ao longo da vida das perturbações de ansiedade comórbidas com a cefaleia em salvas varia entre $4,6 \%$ e $24 \%$ de acordo com os estudos, sendo mais frequentes do que nos casos de cefaleia tipo tensão. ${ }^{13}$ No entanto, na maior parte dos doentes coexiste sintomatologia ansiosa e depressiva.

A comorbilidade entre cefaleias e Perturbação Afectiva Bipolar (PAB) é esperada, na medida em que, ambas as doenças têm uma periodicidade gerada no hipotálamo, relacionam-se com o padrão de sono, partilham alterações neuroendócrinas e respondem eficazmente a terapêuticas específicas, como o lítio. ${ }^{15}$ Apesar de existirem poucos estudos que a demonstrem, segundo Robbins $\mathrm{L},{ }^{16}$ a prevalência de PAB ao longo da vida é de $8,6 \%$ em doentes com enxaqueca crónica, $4,5 \%$ nos casos de cefaleia de tensão crónica e $6,6 \%$ em doentes com cefaleia em salvas crónica. Esta comorbilidade encontra-se sub-diagnosticada, no entanto, estar alerta para este diagnóstico melhora o tratamento dos doentes com cefaleias, na medida em que fármacos como os estabilizadores de humor são pouco utilizados e podiam melhorar quer a PAB quer as cefaleias.

Episódios de comportamentos agressivos, com agitação, são frequentes nos episódios de crises de cefaleia em salvas e alguns estudos de neuroimagem apontam para a sua relação com estruturas cerebrais como o hipotálamo posterior, sugerindo que a neuroestimulação desta área contribui para a melhoria das alterações do comportamento que têm sido correlacionadas com sintomas depressivos e incapacidade.

Pensamentos acerca da morte e ideação suicida podem ocorrer em doentes com cefaleias, nomeadamente nos casos de cefaleia em salvas crónica, correspondendo a $22 \%$ dos doentes, e em $4 \%$ dos casos de enxaqueca. ${ }^{17}$

\section{Mecanismos subjacentes à comorbilidade}

As cefaleias são perturbações que envolvem todo o cérebro, nomeadamente o hipotálamo, estrutura implicada no desenvolvimento da sintomatologia ansiosa e depressiva.

Em estudos de neuroimagem em doentes com cefaleia em salvas, a depressão foi correlacionada com alterações do metabolismo da glicose no córtex insular, e a incapacidade provocada pela dor correlacionou-se com alterações na amígdala, estruturas associadas à patogénese da ansiedade e da depressão. ${ }^{18}$

Além do modelo bidirecional aceite para a comorbilidade entre as perturbações psiquiátricas e as cefaleias, existem factores de risco genéticos e ambientais comuns a ambas as condições que estão implicados na sua génese. Como existe uma expressão familiar significativa quer nas cefaleias, quer em algumas doenças psiquiátricas comórbidas, poderá haver uma relação sindromática entre ambas as condições, mostrada em estudos genéticos. ${ }^{10}$

Factores de risco genéticos e ambientais provocam estados cerebrais latentes que levam à precipitação quer de cefaleias, quer de perturbações psiquiátricas, conforme mostram as evidências neuroimagiológicas. Várias áreas são ativadas durante as crises de cefaleias, como o hipotálamo, a região límbica ou a região cortical. Sinais secundários à dor são conduzidos por terminações trigémino-vasculares para áreas do cérebro que estão envolvidas na produção de sintomas de cefaleias ou depressivos. ${ }^{19}$

Consistentemente com esta hipótese, alguns autores propõem que uma disfunção serotoninérgica e dopaminérgica possa explicar a comorbilidade entre depressão e cefaleias. Níveis alterados de serotonina, diminuição do cortisol sérico e aumento da prolactina são observados durante as crises de cefaleias e frequentemente tratados com triptanos, que são agonistas selectivos dos receptores da serotonina. Estudos experimentais sugerem que pessoas com perturbações de ansiedade e pessoas com cefaleias, nomeadamente enxaquecas, têm polimorfismos comuns no gene transportador de 5-HT. Os receptores de serotonina estão envolvidos na geração do ritmo circadiano a nível do hipotálamo e podem ser um alvo de tratamento farmacológico em doentes com cefaleias, e curiosamente, antagonistas dos receptores de $5 \mathrm{HT}_{7}$ têm sido sugeridos como antidepressivos de ação rápida. A relação entre depressão, ansiedade e disfunção serotoninérgica é bem conhecida, por isso, é razoável especular que a ligação biológica entre cefaleias, perturbações de humor e ansiedade, é a ativação aberrante de neurónios 
no cérebro e no sistema serotoninérgico.

A enxaqueca apresenta tipicamente sintomas dopaminérgicos, como náuseas e vómitos, havendo estudos que mostram níveis elevados de receptores dopaminérgicos nos linfócitos periféricos de pessoas com enxaqueca, sugerindo um estado hipodopaminérgico nestas pessoas. ${ }^{20} \mathrm{~A}$ ligação entre deficiência de dopamina e depressão é bem conhecida e, é possível que a diminuição de dopamina contribua para a comorbilidade entre depressão e cefaleias. Investigadores acreditam que, quer a enxaqueca quer a depressão, possam ser mediadas pela cascata neuronal de eventos que leva à sensibilização central, cuja alodinia cutânea é um marcador, correspondendo a um estado durante o qual os neurónios são anormalmente excitados, levando à redução do limiar da dor e hipersensibilidade a estímulos nocivos e inócuos. Pessoas com enxaqueca e depressão sofrem de alodinia cutânea mais grave do que pessoas com enxaqueca, sem depressão, o que reafirma a hipótese de haver um estado cerebral latente que leva ao desenvolvimento destas duas condições.

A produção de citocinas inflamatórias também pode ser um mecanismo subjacente à comorbilidade entre cefaleias e perturbações psiquiátricas, nomeadamente no caso da $\mathrm{PAB}$, tendo sido mostrados níveis elevados na expressão de várias interleucinas e receptores, que repetidamente libertados durante as crises de cefaleias podem ter implicações no desenvolvimento de sintomatologia depressiva. ${ }^{13}$

Factores de risco ambientais como o consumo de substâncias, traumatismos cranianos, perturbação do padrão de sono e coexistência de enxaqueca, aumentam o risco para o desenvolvimento de crises de cefaleia em salvas. As perturbações do sono, nomeadamente privação do sono ou sono prolongado, podem promover o início de crises de cefaleia, nomeadamente enxaqueca, ${ }^{21}$ no entanto o sono pode ser o factor decisivo para a resolução de uma crise de cefaleia. História de abuso na infância tem sido mostrada como factor de risco para esta comorbilidade, assim como uso frequente de corticóides ou abuso de medicação.

\section{MATERIAL E MÉTODOS}

Os autores efectuaram um estudo descritivo, retrospectivo, de prevalência, envolvendo 250 doentes observados na consulta de ligação de psiquiatria-cefaleias, durante um período de 3 anos, entre 01 de Julho de 2011 e 1 de Julho de 2013, em que se procedeu à análise dos processos clínicos dos doentes observados na Consulta, relativamente aos dados sociodemográficos e clínicos, assim como diagnóstico psiquiátrico, neurológico e terapêutica prescrita.

\section{RESULTADOS}

Dos 250 doentes acompanhados na Consulta, 84\% $(n=210)$ eram do sexo feminino e $16 \%(n=40)$ eram do sexo masculino.

A média de idades foi de 47 anos, situando-se a faixa etária de maior prevalência entre os 41 e 50 anos, variando entre uma idade mínima de 24 até uma idade máxima de 73 anos (Tabela 1).
Tabela 1 - Dados sociodemográficos dos doentes acompanhados na Consulta de Psiquiatria-Cefaleias

\begin{tabular}{ll}
\hline Amostra & $\mathbf{n}=\mathbf{2 5 0}$ \\
\hline Sexo & Sexo feminino: 210 doentes, $84 \%$ \\
& Sexo masculino: 40 doentes, $16 \%$ \\
Idade & Média: 47 anos \\
& $20-30$ anos: $12 \%(n=30)$ \\
& $31-40$ anos: $26 \%(n=65)$ \\
& $41-50$ anos: $38 \%(n=95)$ \\
& $51-60$ anos: $14 \%(n=35)$ \\
& $61-70$ anos: $7,2 \%(n=18)$
\end{tabular}

De acordo com a Classificação Internacional das Cefaleias $-3^{a}$ edição (versão beta), a cefaleia tipo tensão foi a mais prevalente, representando $60,8 \%$ da amostra $(n=$ 152) da consulta de Psiquiatria-Cefaleias (Fig. 1). A enxaqueca correspondeu a $24,8 \%(n=62)$ e as cefaleias atribuídas a perturbações psiquiátricas ocorreram em 18 doentes $(7,2 \%)$. Em menor percentagem estavam também contempladas outras cefaleias secundárias, como a cefaleia em salvas e a nevralgia do trigémio.

Os sintomas psiquiátricos referidos de forma mais comum pelos doentes na Consulta de Psiquiatria de Ligação foram humor deprimido, ansiedade, astenia, insónia e irritabilidade, sendo o humor deprimido $(n=195 ; 78 \%)$,astenia $(n=130 ; 52 \%)$ e insónia $(n=120 ; 48 \%)$ os mais frequentes (Fig. 2). Em termos de diagnósticos psiquiátricos elaborados segundo a CID 10, destacam-se as perturbações de humor (F30-F39), em $62 \%$ dos casos $(n=187)$ e as perturbações neuróticas relacionadas com o stress (F40-F48) em $39,2 \%(n=98)$. Entre as perturbações do humor, as mais prevalentes foram as perturbações depressivas, quer recorrentes, quer episódios atuais (Fig. 3).

As perturbações somatoformes e as perturbações de personalidade também figuravam entre os diagnósticos mais prevalentes nos doentes com cefaleias.

A intervenção terapêutica privilegia o tratamento da sintomatologia ango-depressiva, regularização dos ritmos circadianos e resolução dos paroxismos ansiosos. Para tal, várias estratégias de tratamento são úteis, nomeadamente a psicoeducação, psicoterapia e psicofarmacologia. Entre os psicofármacos mais utilizados neste tipo de doentes 
Outras cefaleias secundárias

Cefaleia atribuída a perturbação psiquiátrica

Nevralgia trigémio

Cefaleia em salvas

Enxaqueca

Cefaleia tipo tensão

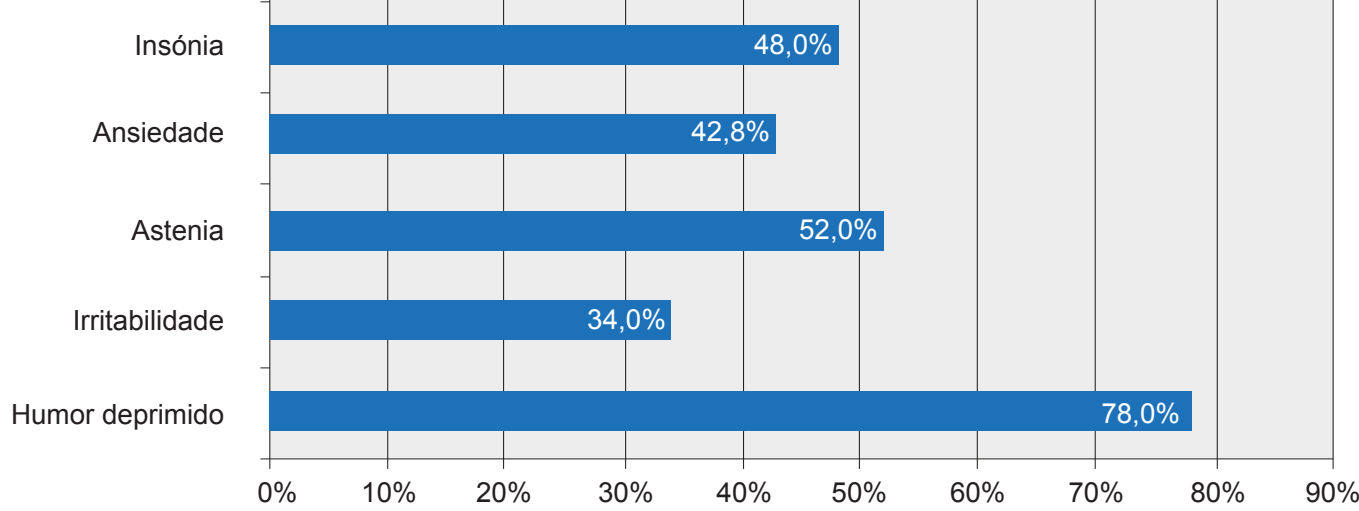

Figura 2 - Sintomas psiquiátricos mais prevalentes na consulta de Psiquiatria-Cefaleias

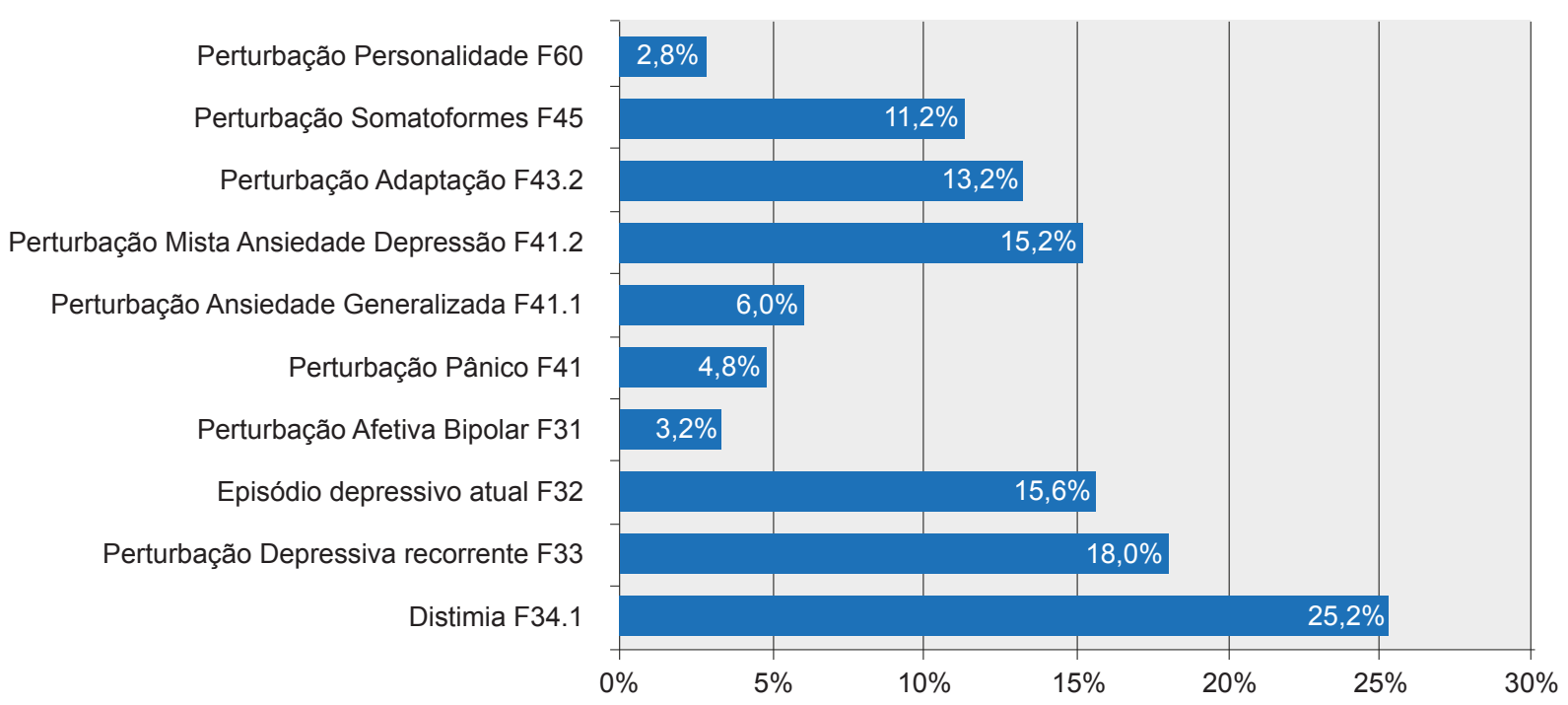

Figura 3 - Diagnósticos psiquiátricos segundo a CID 10 presentes na consulta de Psiquiatria-Cefaleias 
estão os antidepressivos, geralmente da classe dos ISRS, nomeadamente a sertralina e fluoxetina, os antiepilépticos (topiramato) e os ansiolíticos, nomeadamente da classe das benzodiazepinas, que muitas vezes já faziam parte da medicação habitual do doente e eram de difícil retirada (Fig. 4). Cerca de 45 doentes estavam medicados com antidepressivos tricíclicos, como a amitriptilina, e 65 doentes com outros antidepressivos, entre eles, a trazodona.

\section{DISCUSSÃO}

Durante este período de 3 anos foram efectuadas 689 Consultas de Psiquiatria-Cefaleias, verificando-se uma tendência crescente no número de primeiras consultas, encaminhadas maioritariamente através da Consulta Externa de Neurologia.

Após a análise dos dados clínicos, constatou-se que a maioria dos doentes seguidos nesta Consulta eram do sexo feminino (84\%), com média de idades de 47 anos, pertencentes à faixa etária dos $41-50$ anos (38\%), apesar de estarem contemplados todos os grupos etários, desde os 24 aos 73 anos. O tipo mais frequente de cefaleias foram as cefaleias tipo tensão $(60,8 \%)$, a enxaqueca $(24,8 \%)$ e as cefaleias atribuídas a doenças psiquiátricas $(7,2 \%)$, o que está de acordo com a literatura.

A maioria dos doentes apresentava sintomas psiquiátricos de forma comórbida, nomeadamente humor deprimido (78\%), insónia (48\%) e ansiedade $(42,8 \%)$, mas também apresentavam sintomas físicos como a astenia (52\%). Os diagnósticos psiquiátricos mais frequentes foram as perturbações de humor (F30-F39), em $62 \%$ dos casos ( $n=187$ ) e as perturbações neuróticas relacionadas com o stress (F40-F48) em 39,2\% ( $n=98$ ), sendo as perturbações depressivas recorrentes e a distimia as mais frequentes. A presença desta comorbilidade, entre as cefaleias e as perturbações psiquiátricas, complica na maioria das vezes o quadro clínico, aumentando o risco de cronicidade, elevando a intensidade da dor e a taxa de insucesso terapêutico, contribuindo para que as cefaleias constituam uma das principais causas de recurso aos Cuidados de Saúde.

A intervenção terapêutica nestes casos privilegia a intervenção multidisciplinar do médico Neurologista e do Psiquiatra. A psicoeducação e a intervenção psicológica, nomeadamente a abordagem cognitivo-comportamental, são de grande importância nestes doentes. No entanto os psicofármacos desempenham um papel terapêutico em termos profilácticos e no tratamento dos quadros ango-depressivos subjacentes às queixas somáticas de cefaleias. Os antidepressivos, nomeadamente os ISRS, foram os mais usados (76\%), assim como os ansiolíticos da classe das benzodiazepinas (35\%). Os antiepilépticos também mostraram a sua eficácia, principalmente em termos de profilaxia e foram usados em $57 \%$ dos doentes. Foram utilizadas também outras classes de antidepressivos, nomeadamente a trazodona, um antagonista e inibidor da recaptação de serorotina (SARI) e a venlafaxina, um Inibidor da Recaptação de Serotonina e Noradrenalina (IRSN).

\section{CONCLUSÃO}

A comorbilidade entre cefaleias e perturbações psiquiátricas tem implicações importantes na evolução dos quadros clínicos e no tratamento dos doentes afectados por estas condições clínicas, contribuindo para um pior prognóstico e pior qualidade de vida. Após três anos de experiência com a Consulta de Ligação de Psiquiatria-Cefaleias, os autores defendem que o modelo de intervenção multidisciplinar, com intervenção e reconhecimento precoce da presença desta comorbilidade, configura-se uma boa opção no acompanhamento destes doentes, tentando diminuir a complexidade e cronicidade dos quadros clínicos subjacentes.

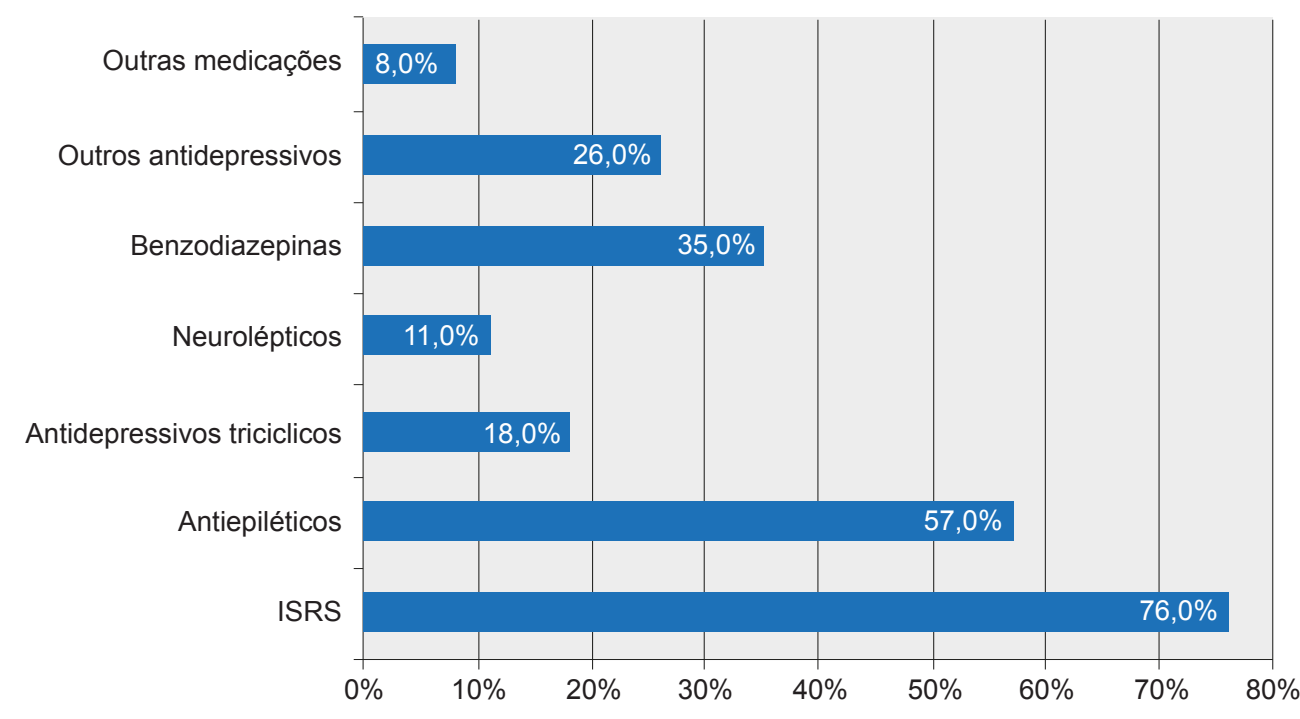

Figura 4 - Tratamento farmacológico prescrito na consulta de Psiquiatria-Cefaleias (ISRS: Inibidores Selectivos da Recaptação de Serotonina). 


\section{CONFLITOS DE INTERESSE}

Os autores declaram que não houve conflitos de interesse nesta investigação.

\section{REFERÊNCIAS}

1. The International Classification of Headache Disorders, 3rd edition (beta version). Cephalalgia. International Headache Society. 2013;33:629 808.

2. Cutrer FM, Black DF. Imaging findings of migraine. Headache. 2006;46:1095-1107.

3. Lipton RB, Silberstein SD. Why study the comorbidity of migraine? Neurology. 1994;44:S4-5.

4. Ashina S, Serrano D, Lipton RB, Maizels M, Manack AN, Turkel CC, et al. Depression and risk of trans- formation of episodic to chronic migraine: results of the American Migraine Prevalence and Prevention study. Enrico Greppi Award 2012. J Headache Pain. 2012;13:615-24.

5. Jette N, Patten S, Williams J, Becker W, Wiebe S. Comorbidity of migraine and psychiatric disorders - a national population-based study. Headache. 2008;48:501-16.

6. Teixeira AL, Costa EA, Júnior AA, Santos IA, Gómez RS, Kummer A, et al. Psychiatric comorbidities of chronic migraine in community and tertiary care clinic samples. J Headaches Pain. 2012;13:551-5.

7. Couch J, Taylor K, Hilliard B. Migraine in the primary care population: prevalence, severity and disability. Headache. 2003;43:570-1.

8. Breslau N, Davis GC, Andreski P. Migraine, psychiatric disorders, and suicide attempts: an epidemiologic study of young adults. Psychiatry Res. 1991;37:11-23.

9. Hamelsky SW, Lipton RB. Psychiatric comorbidity of migraine. Headache. 2006;46:1327-33.

10. Buse DC, Silberstein SD, Manack AN, Papapetropoulos S, Lipton RB. Psychiatric comorbidities of episodic and chronic migraine. J Neurol. 2013;260:1960-9.

11. Manack A, Buse DC, Serrano D, Turkel CC, Lipton RB. Rates, predictors, and consequences of remission from chronic migraine to

\section{FONTES DE FINANCIAMENTO}

Os autores declaram que não houve financiamentos nesta investigação.

episodic migraine. Neurology. 2011;76:711-8.

12. Breslau N, Schultz LR, Stewart WF, Lipton RB, Lucia VC, Welch KM. Headache and major depression: is the association specific to migraine? Neurology. 2000;54:308-13.

13. Robbins MS. The psychiatric comorbidities of cluster headache. Curr Pain Headache Rep. 2013;17:313.

14. Gesztelyi G, Bereczki D. Disability is the major determinant of the severity of depressive symptoms in primary headaches but not in low back pain. Cephalalgia. 2005;25:598-604.

15. Costa A, Leston JA, Cavallini A, Nappi G. Cluster headache and periodic affective illness: common chronobiological features. Funct Neurol. 1998;13:263-72.

16. Robbins L. The bipolar spectrum in migraine, cluster, and chronic tension headache. Eur Neurol Rev. 2008;3:123-4

17. Jürgens TP, Gaul C, Lindwurm A, Dresler T, Paelecke- Habermann Y, Schmidt-Wilcke T, et al. Impairment in episodic and chronic cluster headache. Cephalalgia. 2011; 31:671-82.

18. Seifert $C L$, Valet M, Pfaffenrath V, Boecker $H$, Rüther KV, Tölle TR, et al. Neurometabolic correlates of depression and disability in episodic cluster headache. J Neurol. 2011;258:123-31.

19. Burstein R, Jakubowski M. Neural substrate of depression during migraine. Neurol Sci. 2009;30:S27-31.

20. Barbanti P, Fabbrini G, Ricci A, Pascali MP, Bronzetti E, Amenta F, et al Migraine patients show an increased density of dopamine D3 and D4 receptors on lymphocytes. Cephalalgia. 2000;20:15-19.

21. Bellini B, Arruda M, Cescut A, Saulle C, Persico A, Carotenuto M, et al. Headache and comorbidity in children and adolescents. J Headache Pain. 2013;14:79. 


\section{Comorbilidades Psiquiátricas Associadas às Cefaleias: a Experiência da Consulta de Ligação}

Acta Med Port 2015:28:44-50

Publicado pela Acta Médica Portuguesa, a Revista Científica da Ordem dos Médicos

Av. Almirante Gago Coutinho, 151

1749-084 Lisboa, Portugal.

Tel: +351218428215

E-mail: submissao@actamedicaportuguesa.com

www.actamedicaportuguesa.com

ISSN:0870-399X | e-ISSN: 1646-0758

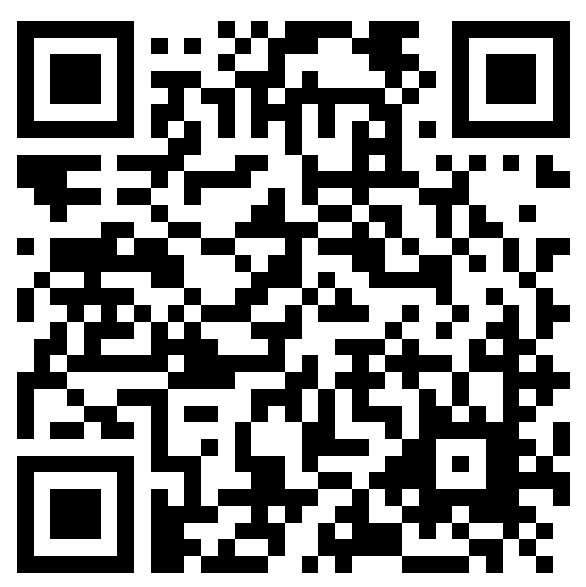

